\title{
Electronic Medical Record and Clinical Evaluation of Two Patient Cohorts at Risk of Hypophosphatasia: Family History is an Underutilized Tool to Diagnose Patients with Rare Disease
}

\section{Christina Peroutka}

University of Virginia https://orcid.org/0000-0001-9122-8550

Natalie M Beck

Genome Medical, Inc.

\section{Ethan Gough}

Johns Hopkins University Bloomberg School of Public Health

Mark Marzinke

Johns Hopkins University School of Medicine

Julie Hoover-Fong ( $\nabla$ jhoover2@jhmi.edu )

Johns Hopkins University https://orcid.org/0000-0002-1242-5626

\section{Research article}

Keywords: Family history, Hypophosphatasia, Electronic Medical Record (EHR), Risk Assessment, Osteoporosis

Posted Date: February 26th, 2021

DOI: https://doi.org/10.21203/rs.3.rs-243884/v1

License: (c) (i) This work is licensed under a Creative Commons Attribution 4.0 International License.

Read Full License 


\section{Abstract}

Background: Family history $(\mathrm{FH})$ is a powerful tool in screening and testing for chronic disease, oncologic conditions, and other genetic diagnoses, and is dependent upon observing patterns of features inherited across generations. Despite acceptance of $\mathrm{FH}$ as a fundamental part of a genetic medicine evaluation, there are limited data in other medical fields about its utility and benefit to patients.

Methods: We evaluated two patient populations at-risk of a rare metabolic condition- hypophosphatasia (HPP) - for the quality and quantity of FH data in their medical records. Population 1 was derived from patients seeking evaluation for a low bone density diagnosis at the Johns Hopkins Greenberg Center for Skeletal Dysplasias (GCSD); population 2 was identified through a targeted electronic medical record (EMR) query of low serum alkaline phosphatase (AP) measurements obtained through the Johns Hopkins Clinical Laboratory.

Results: In population $1(\mathrm{n}=38), 27(71 \%)$ were confirmed to have HPP. Of these, $14(52 \%)$ presented with FH information in the EMR from 3 or more family members, and in 4 (15\%) FH was suggestive of HPP. In population $2(n=348)$, a similar proportion of subjects had 3 or more family members mentioned in the EMR (183 or $53 \%)$, but only 3 subjects ( $1 \%$ included sufficient detail to determine that the family history was suggestive of HPP. Notably, once all patients in population 1 completed a medical genetics evaluation and HPP was confirmed, 20 (74\%) had probable affected family members identified through obtaining and analyzing a pedigree. After cascade testing was offered to these family members, 17 patients $(71 \%)$ from population 1 had at least one family member with HPP confirmed at a molecular level.

Conclusions: Based on these results, we propose that a full genetic medicine evaluation of those subjects from population 2 at highest risk of HPP would confirm HPP in many of them and identify similarly affected family members. We propose that taking a $\mathrm{FH}$ refines the diagnostic precision for patients with low bone density and identifies affected family members, and that HPP is likely more prevalent than previously thought.

\section{Background}

Hypophosphatasia (HPP) is an ultra-rare inherited metabolic condition caused by variants in the tissuenonspecific alkaline phosphatase gene (TNSALP or ALPL, OMIM 171760). There are six forms of disease, with variable age of presentation and severity: perinatal lethal, prenatal benign, infantile onset, childhood/juvenile onset, odontohypophosphatasia and adult-onset. Previous prevalence studies estimate severe HPP affects between 1 in 100,000 and 1 in 400,000 individuals, though population allele frequency suggest a prevalence closer to 1 in 6,370 for all forms(1). Inheritance may be autosomal recessive or dominant, with recessive forms often presenting earlier and with a more severe phenotype. HPP is characterized by pleiotropic manifestations ranging from perinatal lethal bone fragility, respiratory failure and seizures to isolated premature childhood tooth loss or adult-onset musculoskeletal pain, 
fatigue and low bone density(1). Low serum alkaline phosphatase (AP) activity -based on age- and sexspecific norms is a key biochemical indicator of $\operatorname{HPP}(1)$, but is often unrecognized as pathologic. Enzyme replacement therapy (ERT) with asfotase alpha (Strensiq ${ }^{\mathrm{TM}}$ ) was approved by the FDA after demonstration of $97 \%$ survival in a cohort of treated patients with perinatal lethal HPP compared to $42 \%$ survival at 1 year of age in historical controls (2). Misdiagnosis of idiopathic osteoporosis and empiric treatment with bisphosphonates of patients with HPP is problematic since this treatment is contraindicated in HPP patients(3-5). Thus, although HPP is rare compared to idiopathic osteopenia/osteoporosis, individuals with HPP must be accurately diagnosed to receive effective disease-specific treatment and avoid empiric treatment with bisphosphonates, which is associated with significant adverse effects in this population.

Family history (FH) is an integral part of a patient's medical history and diagnostic process. Its importance has been recognized since the beginning of human history, with family histories recorded in the Old Testament and Hippocrates documenting their medical relevance. In current times, FH can be a powerful tool to optimize screening and testing for chronic disease, $(6)$ cancer $(7,8)$ and other conditions, $(7,9-11)$ based on observed patterns of signs and symptoms across generations. FH tools in general medicine and oncology have been shown to have positive analytical validity ( $\mathrm{FH}$ tools measure and obtain the information they are designed to obtain), clinical validity (FH demonstrates significant specificity and sensitivity for disease risk), and clinical utility (FH improves referral, screening and diagnosis rates), without significant negative social implications $(12,13)$.

Despite its clinical value, other literature indicates $\mathrm{FH}$ recorded in the medical record is often incomplete, poorly documented and difficult to access $(9,10,14,15)$. Other barriers to effective utilization of $\mathrm{FH}$ include limited understanding of the genetic basis of conditions, inability to obtain accurate and specific FH from patients, limited time and reimbursement to do so, inadequate tools for obtaining and interpreting the $\mathrm{FH}$, and limited integration and functionality within the electronic medical record $(7,9,10,14-16)$. FH is a fundamental part of the clinical genetic evaluation, and a three-generation pedigree is considered the standard of care.

Until now, there has been no published experience pertaining to the clinical utility of $\mathrm{FH}$ to diagnose individuals with HPP in the genetics clinic. In patients with HPP, a complete FH helps to differentiate HPP from other more common causes of low bone density and bone fragility. Given the spectrum of HPP disease manifestations, the broad variation within affected family members and unappreciated significance of low AP activity measurements, we hypothesize HPP is underdiagnosed. We suspect more patients with HPP can be identified simply by obtaining a $\mathrm{FH}$, and suggest $\mathrm{FH}$ can refine prevalence data for rare disorders, such as HPP. We compared the individual and FHs of patients diagnosed with HPP in our genetics clinic to their own pre-clinical medical records and to the individual and FHs of subjects identified with low AP values in a separate electronic medical record (EMR) query. To our knowledge this is the first study to compare FHs before and after a clinical genetics evaluation in individuals seeking evaluation for a low bone mineral density disease and the $\mathrm{FH}$ of subjects at risk of this rare disease identified through an EMR query. 


\section{Methods}

\section{Study Population}

The $\mathrm{FH}$ recorded in our institutional EMR was examined in two patient populations at risk of a low bone density condition, specifically HPP. The first population was identified from individuals evaluated at the Johns Hopkins Greenberg Center for Skeletal Dysplasias (GCSD) for a potential low bone density condition from April 2008 through March 2020. Each individual underwent a full clinical genetic evaluation, including collection of medical and family history, physical exam, and laboratory screening. Based on these results, individuals determined to have a high probability of HPP underwent molecular testing of the ALPL gene. Of those confirmed to have HPP, FH information recorded in the EMR preceding their initial clinical genetics encounter was also extracted for comparison to that obtained from the clinical genetics encounter.

The second population was identified through a query of all AP levels measured by the Johns Hopkins Hospital Core Laboratories on inpatients and outpatients from January 2013 through December 2015. In collaboration with the Johns Hopkins Center for Clinical Data Analysis (CCDA), the medical record numbers (MRNs) of subjects with one or more low AP levels (see Table 1) were then queried for discrete medical conditions associated with low bone density and low AP (not including HPP). This query included clinic site codes for oncologic treatment (as surrogate for malignancy), those with direct bilirubin $\geq 5 \mathrm{mg} / \mathrm{dL}$ (as surrogate for liver failure), and creatinine $\geq 2.6 \mathrm{mg} / \mathrm{dL}$ (as surrogate for renal failure), and ICD-codes associated with discrete diseases marked by low bone density and hypophosphatasemia (see Appendix A). Subjects identified through this query were excluded from further consideration. Of those remaining, subjects with 5 to 20 serum AP measurements performed over the study interval, of which $\geq 80 \%$ were low based on age- and gender-specific cutoffs (see Table 1 ), underwent a chart review for extraction of all available medical history potentially suggestive of HPP, as well as their FH. All data were abstracted under a waiver of consent. This process was approved by the Johns Hopkins Institutional Review Board.

\section{Data Collection}

Presence or absence of the following FH documentation in the EMR was recorded for each subject in both populations: a three-generation pedigree, family history recorded within the text of medical note(s) and within the EMR FH table. Similarly, the depth of FH documentation was also recorded for the following: at least 1 family member, at least 3 family members and including more than one generation. For patients seen in the GCSD, confirmed FH of HPP was also recorded. Data were collected on uniform data collection forms, and definitions of data fields and a question-by-question (QXQ) document were compiled to ensure consistent data collection. These data were entered into a password-protected Research Electronic Data Capture (REDCap) database on an institutionally-supported server.

\section{Analysis}


Analysis was performed using STATA (v13, StataCorp LP). (17) Summary statistics at the time of data collection of mean age and gender in both populations were tabulated. The proportion with each level and depth of FH documentation was calculated and compared between the 2 populations using chisquare analyses. The FH available for each subject was assessed for consistency with the HPP diagnosis. For the patients seen in the GCSD, the proportion with a FH suggestive of HPP after the clinical genetics evaluation was compared to the proportion who were confirmed to have the diagnosis.

\section{Results}

The first population was identified from the clinical practice of the GCSD from April 2008-March 2020. As shown in figure 1, after clinical assessment, $\mathrm{FH}$, and tailored biochemical assessment of patients presenting for evaluation of a potential low bone density (LBD) condition, 38 probands were considered to have a high likelihood of HPP. In 27 (71\%), molecular testing of ALPL confirmed the diagnosis of HPP; notably, all AP measurements in these individuals were below the age- and sex-specific normal ranges (see Table 1). The FH that was recorded in the EMR prior to the genetic medicine evaluation showed that $63 \%$ had $\mathrm{FH}$ recorded in EMR notes for a minimum of one family member and $52 \%$ had $\mathrm{FH}$ recorded for at least three family members (Table 2). $52 \%$ also had $\mathrm{FH}$ recorded in the FH EMR table and none had a three generation pedigree (increasing to $100 \%$ after clinical evaluation in the GCSD clinic). $15 \%$ had FH suggestive of HPP prior to a clinical evaluation in the GCSD clinic, but this increased to $74 \%$ after the genetic clinical evaluation. After cascade testing of the family members suspected to also have HPP, $63 \%$ of population 1 had at least 1 family member (range 1-3) confirmed at a molecular level as having HPP. Thus from the 27 confirmed probands diagnosed with HPP in our clinic, 17 probands had 28 affected family members.

The second population at risk of hypophosphatasia (HPP) was identified as shown in Figure 2. There were 1,030,121 AP results generated by the Johns Hopkins Hospital (JHH) Core Laboratories from 163,016 inpatient and outpatient individuals over a 3-year period (January 2013 to December 2015). 7,129 individuals were eligible for chart review after exclusion of individuals who had other medical conditions associated with low AP activity measurements (Appendix A). The study population was narrowed further to 348 individuals, based on consistently low AP measurements per the algorithm in Figure 2. All available medical records from these individuals were reviewed for medical history features and FH suggestive of HPP. As shown in Table 2, $85 \%$ of this total population had FH recorded in EMR clinical notes for a minimum of one family member, while $53 \%$ had $\mathrm{FH}$ for three or more family members. $42 \%$ had $\mathrm{FH}$ recorded in the $\mathrm{FH}$ EMR table and $11 \%$ had a three generation pedigree recorded. $1 \% \mathrm{had} \mathrm{FH}$ information recorded in their EMR that was sufficient to suggest a diagnosis of HPP, although all 348 subjects reviewed had recurrent low AP measurements over the study interval.

As shown in Table 2, chi-square analysis showed that there are significantly more individuals in population 2 with a FH reported in at least one relative in the notes than in population $1(p=0.007989)$. There is no difference in the proportion with a $\mathrm{FH}$ in at least one relative in the EMR table in population 1 as compared to population $2(p=0.316477)$. Similarly, there is no difference in the proportion with a FH 
for at least three relatives (including all possible sections - notes and/or the EMR table) in population 1 as compared to population $2(\mathrm{p}=0.941319)$. There are significantly more individuals in population 1 with a FH suggestive of HPP than in population 2 prior to clinical evaluation $(p<0.00001)$.

\section{Discussion}

Family history can identify individuals at risk of heritable rare diseases as well as identify affected family members who may also benefit from medical management. However, review of patients followed in our clinical center (population 1) and the results of our retrospective EMR study (population 2) indicate that $\mathrm{FH}$ is underutilized in clinical practice. This study began with the observation that most individuals diagnosed with HPP in our clinical center (population 1) had affected family members. These family members were identified for further testing only after collection of a 3 generation pedigree. This difference before and after collection of a comprehensive $\mathrm{FH}$ highlights its underutilization in medical practice.

Population 2, as outlined in Figure 2, involved an unbiased sampling of AP measurements in all patients evaluated over a 3 year period at our institution. After enriching the population for those at greatest risk of HPP and performing a comprehensive chart review of these remaining subjects, we found that the FH data available in the EMR were insufficient in most cases to assess the likelihood that their relatives had a low bone density condition that could be considered with the proband's history. The quantity of FH recorded in the EMR for population 2 showed comparable results to that of population 1. Less than half of individuals had FH recorded in the EMR FH table, the most readily available location in the EMR for healthcare providers to access and utilize these data. Most importantly, only $1 \%$ of this enriched population had sufficient detail in the recorded $\mathrm{FH}$ to allow for identification of a possible genetic skeletal condition in a relative. Even with chronically and clinically significantly low serum AP values, few providers ask disease-directed questions about family members.

In this study, FH was often mentioned in a clinical visit or note, but often included information about only 1 or 2 relatives and was difficult to access in the EMR or share among providers. We also found it was more common for $\mathrm{FH}$ to be recorded in notes versus in the $\mathrm{FH}$ table of the EMR, even though the $\mathrm{FH}$ table is more accessible during clinic appointments to other providers. At our institution, the structure of the $\mathrm{FH}$ table does not allow for visualization of $\mathrm{FH}$ across generations in the same way that a pedigree does. Some EMRs allow for pedigree creation from a FH table, but this function was not available at our institution when the study was performed. Additionally, pedigrees and other documents are catalogued within sections of the EMR which may be less accessible to providers at the point of care and unsearchable for recorded details of the FH. Specifically for the EMR study of population 2, a limitation of the EMR query was the inability to query by 'text search' in notes, $\mathrm{FH}$ table, or scanned documents. All data on the 348 charts were manually read and reviewed. It is possible that HPP was considered for additional individuals within the 7,129 patients with unexplained low serum AP, but who did not meet our refined criteria of 5 to 20 AP activity measurements performed over the study interval, of which $\geq 80 \%$ were low. 
Another limitation of the current study is that lineage or side of the family was not a qualifier to include/exclude FH data. In the EMR population (population 2), we assumed all affected family members were from the same side of the family, thereby increasing risk of HPP. When assessed in-person, this may not actually be correct. In our description of these study cohorts (manuscript in preparation), $17 \%$ of population 2 were considered at-risk of HPP, but only $1 \%$ agreed to a clinical research evaluation. It is therefore not possible to know how many are actually affected with HPP and have affected family members. While the population seeking care at the GCSD (population 1) is a biased cohort of individuals who are referred due to concerns for bone disease, the data from this cohort underscore that in-person assessments including a three-generation pedigree adds substantially to the $\mathrm{FH}$ data available in the medical record, allows for identification of affected family members, and potentially refines disease prevalence data, especially for rare disease.

Research on $\mathrm{FH}$ to date suggests that publically accessible tools that generate a multigenerational $\mathrm{FH}$ prior to a clinic appointment help the provider to identify, screen and care for individuals with rare disease. $(10,18)$ Our study highlights that 'rare' diseases may not be so rare if family history is collected and utilized in clinical practice. We designed a FH and risk-stratification tool for individuals with low bone mineral density that includes guidance for patients and providers based on other signs and symptoms, including serum AP levels (bonegenetics.org). After IRB approval, this research tool will refine the signs and symptoms that are the strongest predictors of HPP. Increased recognition of HPP is essential as the treatment of those patients varies from standard therapy for low bone density and bone fragility.

\section{Conclusions}

Our study demonstrates that when a three-generation pedigree is obtained, more disease-specific evaluations can be pursued, more accurate diagnoses can be made, and affected family members are brought to medical attention through appropriate family risk counseling and screening. Although HPP is defined as an ultra-rare condition, the key features of bone fragility and decreased BMD overlap with osteopenia/osteoporosis, which is relatively common. In this study, when a three-generation $\mathrm{FH}$ and appropriate testing were completed, 27 patients were diagnosed with HPP in our center and 28 additional family members were also able to be diagnosed with HPP. This represents a doubling of disease prevalence of individuals diagnosed with this HPP, simply by taking a FH suggesting that HPP may not be ultra-rare. Despite the promising illustration of $\mathrm{FH}$ utilization within our clinical practice, challenges remain in applying EMR query of large data to identify individuals at-risk of inherited rare diseases, such as HPP, within a health system, especially when identification relies on FH documentation within the EMR.

\section{List Of Abbreviations}

Alkaline phosphatase (AP)

Center for Clinical Data Analysis (CCDA) 
Electronic medical record (EMR)

Enzyme replacement therapy (ERT)

Family history (FH)

Greenberg Center for Skeletal Dysplasias (GCSD)

Hypophosphatasia (HPP)

Low bone density (LBD)

Medical record numbers (MRNs)

Question-by-question (QXQ)

Research Electronic Data Capture (REDCap)

\section{Declarations}

Ethics approval and consent to participate: This study was approved by the Johns Hopkins Institutional Review Board (IRB) Study 00066333 under a waiver of consent.

Consent for publication:- not applicable

Availability of data and materials: The datasets generated and/or analyzed during the current study are not publicly available due to being protected health information (PHI) but are presented in aggregate as was approved by the Johns Hopkins Data Trust Committee.

\section{Competing interests: none}

Funding: The retrospective chart review was partially funded by Alexion Pharmaceuticals $₫$ and the Greenberg Center for Skeletal Dysplasias. This funding covered the costs for data extraction from our institutional EMR and for labor for the retrospective chart review.

Authors' contributions: $\mathrm{CP}$ and JHF completed the retrospective chart review. NB, CP and JHF completed the chart review of GCSD patients. MM completed the AP measurement query. EG assisted with the statistics.

Acknowledgements: We acknowledge Natasha Parikh, research assistant in the Greenberg Center for Skeletal Dysplasias, for data entry.

Authors' information (optional): see above.

\section{References}


1. Mornet E. Hypophosphatasia. Metabolism. 2018 May 1;82:142-55.

2. StrensiqTM (asfotase alfa) FDA-approved package insert [Internet]. [cited 2020 Aug 19]. Available from: https://www.accessdata.fda.gov/drugsatfda_docs/label/2015/125513s000lbl.pdf

3. Whyte MP. Atypical Femoral Fractures, Bisphosphonates, and Adult Hypophosphatasia. Journal of Bone and Mineral Research. 2009;24(6):1132-4.

4. Sutton RA, Mumm S, Coburn SP, Ericson KL, Whyte MP. "Atypical femoral fractures" during bisphosphonate exposure in adult hypophosphatasia. Journal of Bone and Mineral Research. 2012;27(5):987-94.

5. Cundy T, Michigami T, Tachikawa K, Dray M, Collins JF, Paschalis EP, et al. Reversible Deterioration in Hypophosphatasia Caused by Renal Failure With Bisphosphonate Treatment. Journal of Bone and Mineral Research. 2015;30(9):1726-37.

6. Carroll JC, Campbell-Scherer D, Permaul JA, Myers J, Manca DP, Meaney C, et al. Assessing family history of chronic disease in primary care. Canadian Family Physician. 2017 Jan;63:10.

7. Cleophat JE, Nabi H, Pelletier S, Bouchard K, Dorval M. What characterizes cancer family history collection tools? A critical literature review. Current Oncology [Internet]. 2018 Aug 22 [cited 2019 May 23];25(4). Available from: https://current-oncology.com/index.php/oncology/article/view/4042

8. Orlando LA, Wu RR, Myers RA, Buchanan AH, Henrich VC, Hauser ER, et al. Clinical utility of a Webenabled risk-assessment and clinical decision support program. Genetics in Medicine. 2016 Oct;18(10):1020-8.

9. Berg AO, Baird MA, Botkin JR, Driscoll DA, Fishman PA, Guarino PD, et al. NIH Consensus and Stateof-the-Science Statements: Family History and Improving Health. 2009;26(1):35.

10. de Hoog CLMM, Portegijs PJM, Stoffers HEJH. Family history tools for primary care are not ready yet to be implemented. A systematic review. European Journal of General Practice. 2014 Jun;20(2):12533.

11. Houwink EJF, Hortensius OR, van Boven K, Sollie A, Numans ME. Genetics in primary care: validating a tool to pre-symptomatically assess common disease risk using an Australian questionnaire on family history. Clinical and Translational Medicine. 2019 May 2;8(1):17.

12. ACCE Model Process for Evaluating Genetic Tests I CDC [Internet]. 2019 [cited 2019 Jun 19]. Available from: https://www.cdc.gov/genomics/gtesting/acce/index.htm

13. ACCE Model List of 44 Targeted Questions Aimed at a Comprehensive Review of Genetic Testing I CDC [Internet]. 2019 [cited 2019 Jun 19]. Available from:

https://www.cdc.gov/genomics/gtesting/acce/acce_proj.htm

14. Pyeritz RE. The family history: the first genetic test, and still useful after all those years? Genetics in Medicine. 2012 Jan;14(1):3-9.

15. Orlando LA, Hauser ER, Christianson C, Powell KP, Buchanan AH, Chesnut B, et al. Protocol for implementation of family health history collection and decision support into primary care using a computerized family health history system. BMC Health Serv Res. 2011 Oct 11;11:264. 
16. Feero WG. Connecting the Dots Between Patient-Completed Family Health History and the Electronic Health Record. Journal of General Internal Medicine. 2013 Dec;28(12):1547-8.

17. StataCorp. 2015. Stata Statistical Software: Release 14. College Station, TX: StataCorp LP.

18. Taylor DP, Taylor DP, Hulse NC, Hulse NC, Wood GM, Haug PJ, et al. Ideal features for a patiententered family history and risk assessment tool. AMIA Annu Symp Proc. 2008 Nov 6;1152.

19. Alkaline Phosphatase Isoenzymes, Serum or Plasma | ARUP Lab Test Directory [Internet]. [cited 2020 Oct 28]. Available from: https://ltd.aruplab.com/Tests/Pub/0021020

20. Colantonio DA, Kyriakopoulou L, Chan MK, Daly CH, Brinc D, Venner AA, et al. Closing the gaps in pediatric laboratory reference intervals: a CALIPER database of 40 biochemical markers in a healthy and multiethnic population of children. Clin Chem. 2012 May;58(5):854-68.

\section{Tables}

Table 1 - Lowest Normal Total Serum or Plasma Alkaline Phosphatase (AP) Activity (U/L) Adapted from ARUP Laboratories and Colantonio et al 2012. $(19,20)$

\begin{tabular}{|lll|}
\hline \multirow{2}{*}{ Age } & \multicolumn{2}{l|}{ Lowest Normal Total Serum or Plasma Alkaline Phosphatase Activity (U/L) } \\
\cline { 2 - 4 } & Male & Female \\
\hline 0-30 days & 60 & 60 \\
\hline $1-11$ months & 70 & 70 \\
\hline $1-3$ years & 125 & 125 \\
\hline $4-11$ years & 150 & 150 \\
\hline $12-13$ years & 160 & 110 \\
\hline $14-15$ years & 130 & 55 \\
\hline $16-19$ years & 60 & 40 \\
\hline$>20$ years & 40 & 40 \\
\hline
\end{tabular}

Table 2 - Level and Depth of Family History Documentation Population \#1 includes patients seeking a clinical genetic evaluation at the GCSD. Population \#2 includes subjects identified through EMR query. Family history documentation level (presence or absence) and depth (number of relatives documented) was recorded for each individual in both populations. 


\begin{tabular}{|c|c|c|c|}
\hline & $\begin{array}{l}\text { Population } \\
\# 1\end{array}$ & $\begin{array}{l}\text { Population } \\
\text { \#2 }\end{array}$ & $\begin{array}{l}\text { Population \#1 vs. } \\
\# 2\end{array}$ \\
\hline & $\mathrm{N}=\mathbf{2 7}$ & $N=348$ & Chi-square test \\
\hline & & & $P$ value \\
\hline & $\mathbf{N}(\%)$ & $N(\%)$ & \\
\hline Family history for $\geq 1$ relative in notes & $17(63)$ & $295(85)$ & 8.5247 \\
\hline & & & $P=0.007989$ \\
\hline Family history for $\geq 1$ relative in EMR table & $14(52)$ & $146(42)$ & 1.0034 \\
\hline & & & $P=0.316477$ \\
\hline Family history for $\geq 3$ relatives in notes or & $14(52)$ & $183(53)$ & 0.0054 \\
\hline & & & $P=0.941319$ \\
\hline 3-generation pedigree & $0(0)$ & $37(11)$ & NA \\
\hline Family history suggested HPP in clinical & $4(15)$ & $3(1)$ & 50.6318 \\
\hline & & & $P<0.00001$ \\
\hline
\end{tabular}

$X^{2}($ degrees of freedom, $N=$ sample size $)=$ chi-squared statistic value, $p=p$ value.

\section{Figures}


38 probands presented to the GCSD for a clinical genetics evaluation for a potential metabolic bone disease and assessed to be at-risk of HPP

ALPL sequencing, deletion and duplication analysis confirms a molecular diagnosis of HPP in $27 / 38(71 \%)$

27 probands with a confirmed molecular diagnosis of HPP undergo chart review for family history data (see Table 2) and a 3-generation pedigree is obtained for all

$4(15 \%)$ probands have a family history suggestive of HPP on pre-clinic review

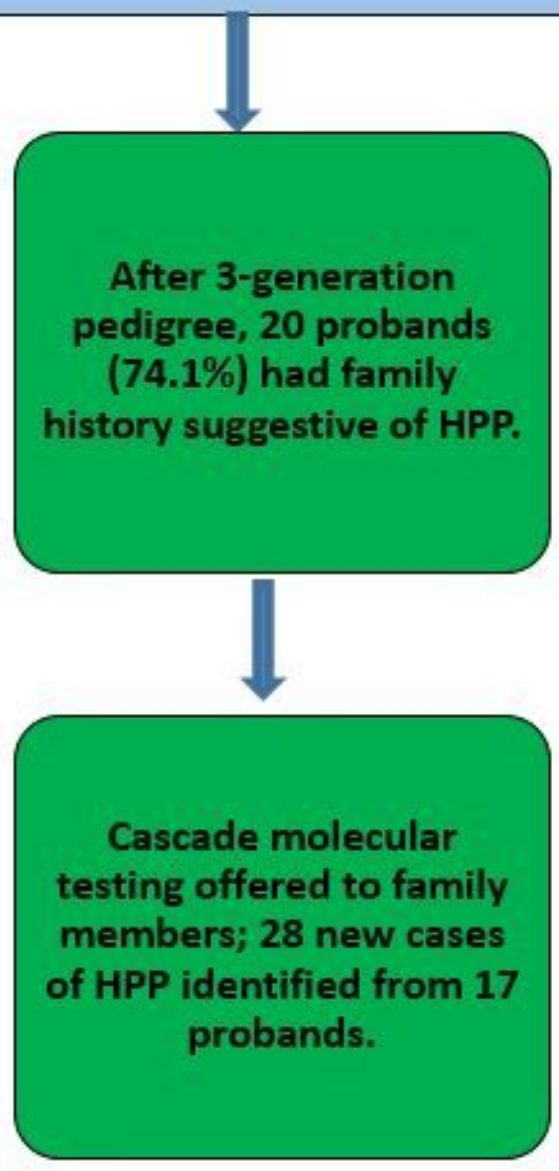

\section{Figure 1}

Algorithm used to evaluate patients presenting to the GCSD for potential metabolic bone disease then diagnosed with HPP from 4/2008-3/2020 
$1,030,121$ AP measurements from 163,016 subjects from $1 / 2013$ through $12 / 2015$

$\mathbf{9 8 3 , 4 5 8}$ observations from 156,459 subjects after exclusion of cancelled AP results or incomplete demographic data

45,233 low measurements $(22,175$ in subjects < 19 years; 23,058 in subjects $\geq 19$ years) from $\mathbf{1 1 , 7 3 0}$ subjects (all ages) using age- and sex-specific AP references

7,129 subjects eligible for chart review after exclusion of subjects with ICD- codes associated with low AP levels, site codes, and liver or renal failure.

Assume a subject with $\geq 5$ to 20 AP levels over 3 years that are $80 \%$ abnormal may have HPP $\rightarrow \mathbf{3 4 8}$ subjects eligible for chart review (157 with $100 \%$ abnormal AP)

348 charts reviewed for family history data (see Table 2).

3 subjects have family history suggestive of HPP

Figure 2

Algorithm used to identify individuals at risk of HPP through an EMR query

\section{Supplementary Files}

This is a list of supplementary files associated with this preprint. Click to download. 
- appendixA.docx

Page $14 / 14$ 